\title{
New insights into genetic susceptibility of COVID-19: an ACE2 and TMPRSS2 polymorphism analysis
}

Yuan Hou', Junfei Zhao ${ }^{2}$, William Martin ${ }^{1}$, Asha Kallianpur ${ }^{1,3}$, Mina K. Chung ${ }^{3,4,5}$, Lara Jehi ${ }^{5}$, Nima Sharifi, Serpil Erzurum ${ }^{3,5}$, Charis Eng ${ }^{1,3,6,7}$ and Feixiong Cheng ${ }^{1,3,7^{*}}$

\begin{abstract}
Background: Coronavirus Disease 2019 (COVID-19), caused by the severe acute respiratory syndrome coronavirus 2 (SARS-CoV-2), has now been confirmed worldwide. Yet, COVID-19 is strangely and tragically selective. Morbidity and mortality due to COVID19 rise dramatically with age and co-existing health conditions, including cancer and cardiovascular diseases. Human genetic factors may contribute to the extremely high transmissibility of SARS-CoV-2 and to the relentlessly progressive disease observed in a small but significant proportion of infected individuals, but these factors are largely unknown.

Main body: In this study, we investigated genetic susceptibility to COVID-19 by examining DNA polymorphisms in ACE2 and TMPRSS2 (two key host factors of SARS-CoV-2) from 81,000 human genomes. We found unique genetic susceptibility across different populations in ACE2 and TMPRSS2. Specifically, ACE2 polymorphisms were found to be associated with cardiovascular and pulmonary conditions by altering the angiotensinogen-ACE2 interactions, such as p.Arg514Gly in the African/African-American population. Unique but prevalent polymorphisms (including p.Val160Met (rs12329760), an expression quantitative trait locus (eQTL)) in TMPRSS2, offer potential explanations for differential genetic susceptibility to COVID-19 as well as for risk factors, including those with cancer and the highrisk group of male patients. We further discussed that polymorphisms in ACE2 or TMPRSS2 could guide effective treatments (i.e., hydroxychloroquine and camostat) for COVID-19.
\end{abstract}

Conclusion: This study suggested that ACE2 or TMPRSS2 DNA polymorphisms were likely associated with genetic susceptibility of COVID-19, which calls for a human genetics initiative for fighting the COVID-19 pandemic.

Keywords: Angiotensin-converting enzyme 2 (ACE2), Coronavirus, COVID-19, Genetic susceptibility, SARS-CoV-2, TMPRSS2

\footnotetext{
* Correspondence: chengf@ccf.org

${ }^{1}$ Genomic Medicine Institute, Lerner Research Institute, Cleveland Clinic, Cleveland, OH 44195, USA

${ }^{3}$ Department of Molecular Medicine, Cleveland Clinic Lerner College of Medicine, Case Western Reserve University, Cleveland, OH 44195, USA

Full list of author information is available at the end of the article
}

(c) The Author(s). 2020 Open Access This article is licensed under a Creative Commons Attribution 4.0 International License, which permits use, sharing, adaptation, distribution and reproduction in any medium or format, as long as you give appropriate credit to the original author(s) and the source, provide a link to the Creative Commons licence, and indicate if changes were made. The images or other third party material in this article are included in the article's Creative Commons licence, unless indicated otherwise in a credit line to the material. If material is not included in the article's Creative Commons licence and your intended use is not permitted by statutory regulation or exceeds the permitted use, you will need to obtain permission directly from the copyright holder. To view a copy of this licence, visit http://creativecommons.org/licenses/by/4.0/. The Creative Commons Public Domain Dedication waiver (http://creativecommons.org/publicdomain/zero/1.0/) applies to the data made available in this article, unless otherwise stated in a credit line to the data. 


\section{Background}

Coronaviruses (CoVs), so named for their "crown-like" appearance by electron microscopy, are a large family of viruses that spread from animal hosts (e.g., bats, civet, cats) to humans, causing life-threatening respiratory diseases like Middle East respiratory syndrome (MERS) and severe acute respiratory syndrome (SARS) [1]. As of June 18, 2020, over 8.4 million cases and 450,000 deaths resulting from infection by a novel SARS coronavirus, SARS-CoV-2 (also termed Coronavirus Disease 2019 or COVID-19), have now been confirmed worldwide; furthermore, there have been more than 2.2 million confirmed cases and over 110,000 deaths due to the COVID-19 pandemic in the USA alone [2]. Unlike other CoVs, SARS-CoV-2 has had much larger global spread and has impacted more people than SARS-CoV-1 and MERS-CoV combined [1]. Morbidity and mortality due to COVID-19 rise dramatically with age and co-existing health conditions, including cancer and cardiovascular diseases, and while most infected individuals recover, even very young and otherwise healthy patients may unpredictably succumb to this disease [3]. These observations beg the question of how much of the variation in COVID-19 disease severity may be explained by genetic susceptibility. Human genetic factors may contribute to the extremely high transmissibility of SARS-CoV-2 and to the relentlessly progressive disease observed in a small but significant proportion of infected individuals; yet, these factors are largely unknown. Development of new preventive and/or therapeutic strategies for COVID-19 will be greatly facilitated by systematic identification of host genetic pathways and DNA polymorphisms (variants) which modulate the risk of infection and severe illness, including the overexuberant immune response to the virus that often portends a poor outcome.

Not only has the COVID-19 pandemic had huge health and economic impacts in 188 countries/regions across the world, but the disease has also struck in different racial/ethnic subpopulations. Large genetic studies in populations of geographically diverse ancestry have demonstrated substantial genetic variation in proteincoding regions, with widely varying allele frequencies [4]. SARS-CoV-2 infection depends on the host cell factors angiotensin-converting enzyme 2 (ACE2) for entry into cells and the host transmembrane serine protease TMPRSS2 for SARS-CoV-2 spike (S) protein priming [5] (Fig. 1a). ACE2, encoded on the X-chromosome, catalyzes the conversion of angiotensin II to angiotensin-(1$7)$, which acts as a vasodilator and exerts important modulatory effects on the cardiovascular system. TMPRSS2 is a key gene in prostate cancer, as an associated translocation drives ETS-family oncogene expression in a large proportion of tumors [6]. The distribution of ACE2 expression has recently been investigated by single-cell RNA sequencing, and the expression of both ACE2 and TMPRSS2 are likely to dictate SARS-CoV-2 tissue tropism [7]. Clinical studies have reported that incidence and mortality rates are significantly different between male and female COVID-19 patients, and the disease is associated with pre-existing conditions, such as cancer and cardiovascular disorders, in particular individuals with hypertension receiving anti-hypertensive medications [8]. Therefore, a systematic investigation of the functional polymorphisms in ACE2 and TMPRSS2 among different populations could pave the way for precision medicine and personalized treatment strategies for COVID-19.

\section{ACE2 polymorphism analysis across different populations}

Here, we investigated genetic susceptibility to COVID19 by examining DNA polymorphisms in ACE2 (OMIM 300335) and TMPRSS2 (OMIM 602060) genes. We assembled a total of 437 non-synonymous singlenucleotide variants (SNVs) in the protein-coding regions of ACE2 and TMPRSS2 (Fig. 1a) from three databases: (i) Genome Aggregation Database (gnomAD v3: gnomad.broadinstitute.org, covering 9 geographical areas), (ii) Exome Sequencing Project (ESP: evs.gs.washington. edu/EVS/), and (iii) 1000 Genomes Project (1KGP, www. internationalgenome.org). We used ANNOVAR [9] to annotate all non-synonymous variants. By applying Polyphen2 and CADD (Combined Annotation Dependent Depletion) scores, we identified 63 potentially deleterious variants in ACE2 (61 in gnomAD) and 68 deleterious variants in TMPRSS2 (63 in gnomAD).

We found that the distribution of deleterious variants in ACE2 differs among 9 populations in gnomAD (v3). Specifically, 39\% (24/61) and 54\% (33/61) of deleterious variants in ACE2 occur in African/African-American (AFR) and Non-Finnish European (EUR) populations, respectively (Fig. 1b). Prevalence of deleterious variants among Latino/Admixed American (AMR), East Asian (EAS), Finnish (FIN), and South Asian (SAS) populations is $2-10 \%$, while Amish (AMI) and Ashkenazi Jewish (ASJ) populations do not appear to carry such variants in ACE2 coding regions (Fig. 1b). Specifically, several variants, including p.Met383Thr, p.Pro389His, and p.Asp427Tyr, have been reported to slightly inhibit the interaction between ACE2 and the spike protein of SARS-CoV-1 [10], which caused the first global SARSCoV-1 outbreak. Only AFR populations carry p.Met383Thr and p.Asp427Tyr variants, with allele frequencies of $0.003 \%$ and $0.01 \%$, respectively (Fig. 1b). The p.Pro389His only occurs in the AMR populations, with an allele frequency of $0.015 \%$. The p.Arg514Gly is a low allele frequency $(0.003 \%)$ variant in AFR populations and is also somatically mutated in colon cancers and 


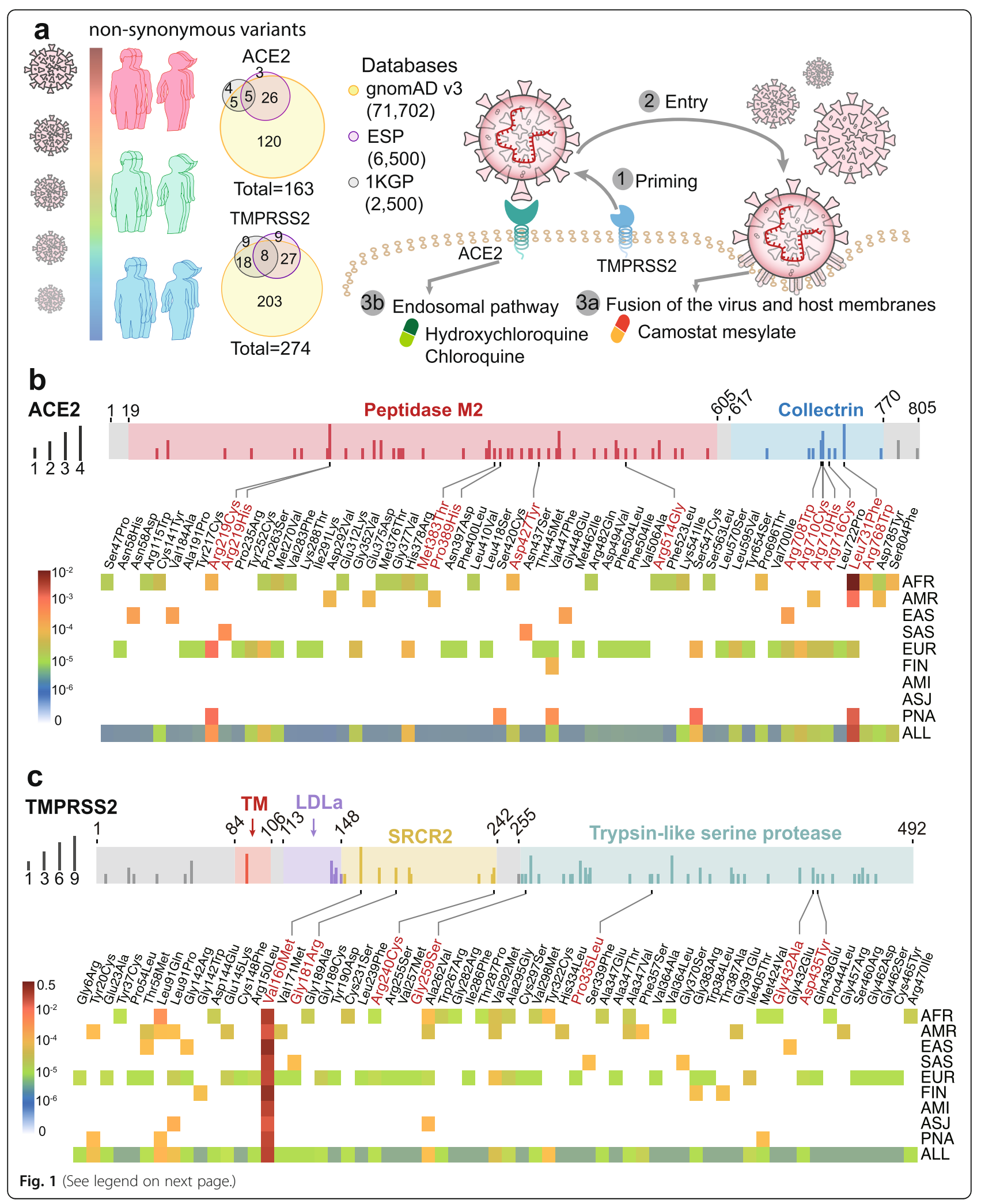




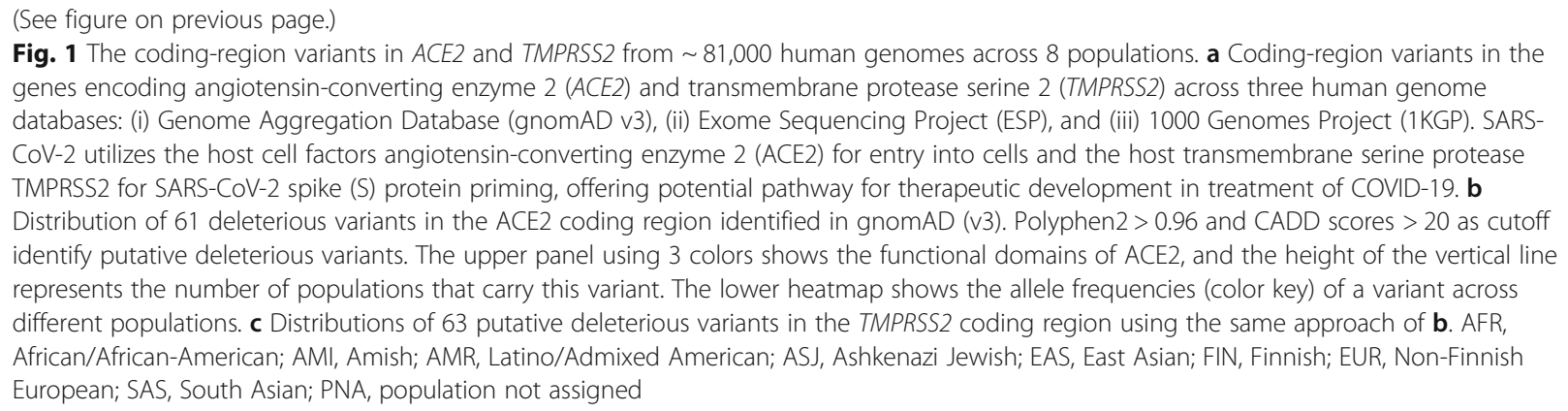

melanomas from The Cancer Genome Atlas (TCGA: https://portal.gdc.cancer.gov). This ACE2 variant is located in the angiotensinogen (AGT)-ACE2 interaction surface, which is anticipated to influence the reninangiotensin system (RAS) function. The RAS is critical for regulation of blood pressure, sodium, and fluid balance, and its dysfunction is associated with cardiovascular and kidney disorders [11]. Residues Arg708/710/716 are located in the dimeric interface of ACE2 (Fig. 2a), and they are essential for its cleavage by TMPRSS2; this processing is required for augmentation of SARS-Sdriven entry into host cells [12]. The EUR population carries the p.Arg708Trp, p.Arg710Cys, p. Arg710His, and p.Arg716Cys variants with allele frequency of $0.01 \sim 0.006 \%$ (Fig. 1a), while the EAS and the AMR populations only carry p.Arg708Trp and p.Arg710His with allele frequency of $0.04 \%$ and $0.01 \%$ respectively. In addition to these four variants, p.Leu731Phe has the highest allele frequency in the AFR and EUR populations. We further inspected the expression quantitative trait loci (eQTL) for ACE2 using the GTEx [13] and QTLbase [14] databases. We did not find any eQTLs for $A C E 2$ from the GTEx, while we found one weak eQTL associated with ACE2 non-synonymous SNP (rs41303171) in the kidney from the QTLbase [14].

Altogether, these comparative genetic analyses suggest that $A C E 2$ genomic variants may play important roles in susceptibilities to COVID-19 and its associated cardiovascular conditions by altering AGT-ACE2 pathway (i.e., p.Arg514Gly). In addition to differential polymorphisms which may explain susceptibility and even outcome in different ethnic populations, the fact that ACE2 is localized to Xp22.2 may help explain the observed maleassociated risk. As such, even in the absence of variation in this gene, the monoallelic versus biallelic presence of this gene may impact the natural history and prognosis of COVID-19 in males.

\section{TMPRSS2 polymorphism analysis across different populations}

TMPRSS2 enzyme activity is important for coronavirus spread and pathogenesis in the infected host [15]. Our analysis indicates $4 \%(11 / 274)$ of non-synonymous variants of TMPRSS2 are stop-gained mutations and carried by AFR and EUR with low allele frequency $(7.0 \times$ $\left.10^{-6} \sim 1.4 \times 10^{-5}\right)$. Meanwhile, 35\% (22/63) and 59\% (37/ 63) of deleterious variants in TMPRSS2 coding regions are carried by the AFR and EUR populations from gnomAD (v3), respectively (Fig. 1c). Each of the EAS, SAS, and FIN populations only carries 4 deleterious variants. We found 6 germline deleterious variants (p.Val160Met, p.Gly181Arg, p.Arg240Cys, p.Gly259Ser, p.Pro335Leu, and p.Gly432Ala) in the TMPRSS2 coding region, which are also identified as somatic mutations occurring in different cancer types from TCGA and COSMIC databases (https://cancer.sanger.ac.uk/cosmic).

We further evaluated the eQTL profile of TMPRSS2 using the GTEx [13] and QTLbase databases [14] as well. We found two eQTLs associated with TMPRSS2 non-synonymous SNPs (rs12329760 (encoding p.Val160Met), $p=4.54 \times 10^{-5}$; rs75603675, $\left.p=0.009\right)$ in the kidney and bone, respectively, using the QTLbase database [14], while there are no known eQTLs associated with TMPRSS2 non-synonymous SNPs from GTEx [13]. Notably, all populations carry p.Val160Met variants with the highest allele frequency $(\sim 25 \%)$, especially for the EAS population at a $40 \%$ allele frequency. Asp435 is a key residue for catalytic substrate binding of TMPRSS2 (Fig. 2b). We found that the p.Asp435Tyr, which has low allele frequency, is carried by the EUR population only (Fig. 1c). These unique but prevalent polymorphisms in TMPRSS2 offer potential explanations for differential genetic susceptibility to COVID-19 as well as for risk factors, including those with cancer and the high-risk group of male patients. Because TMPRSS2 is located on 21q22.3, we could speculate that individuals with Down syndrome would be at high risk for COVID19 infection. In addition, oncogenic roles of TMPRSS2 may be linked to poor outcomes with COVID-19 as well [16], which should be studied in the future. Using single-cell RNA-sequencing analysis, Schuler et al. showed that TMPRSS 2 expression was highest in ciliated cells and type I alveolar epithelial cells (AT1) and increased with aging in humans and mice [17]. This 


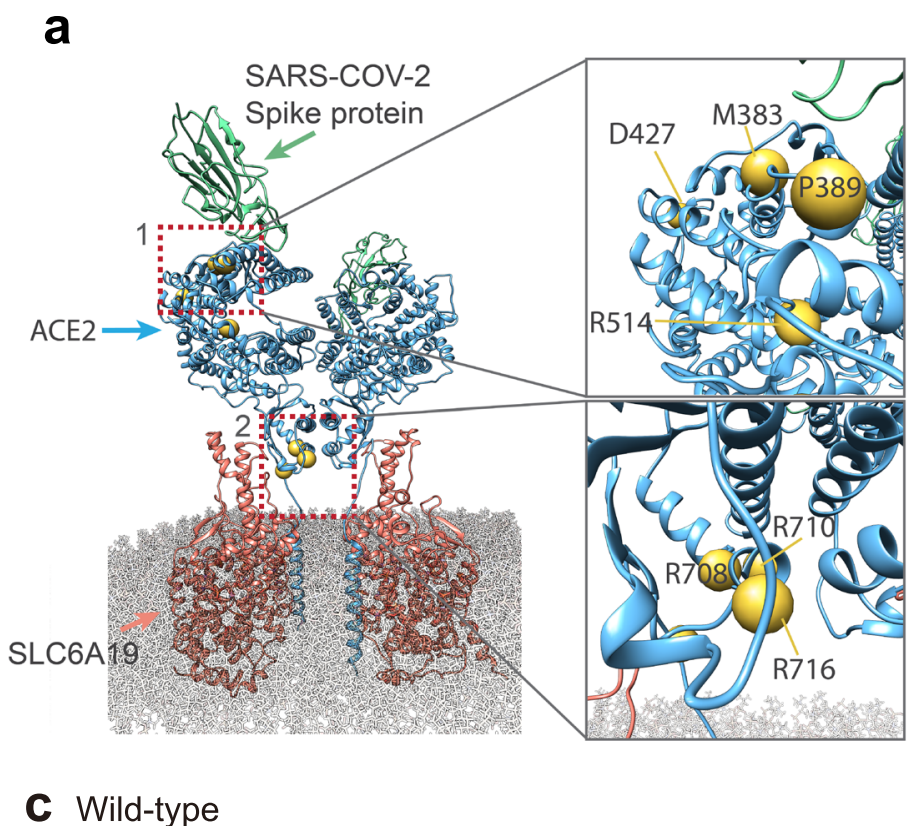

b

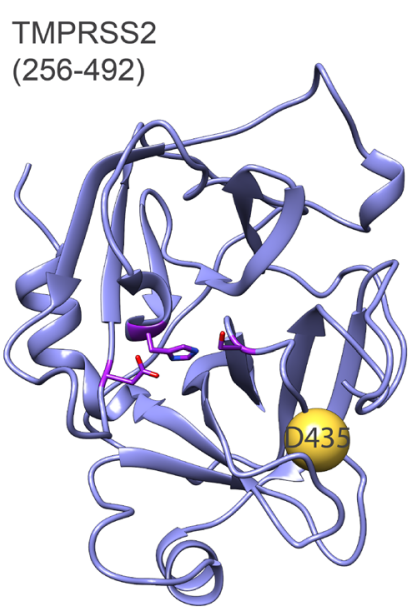

Therapeutic options:
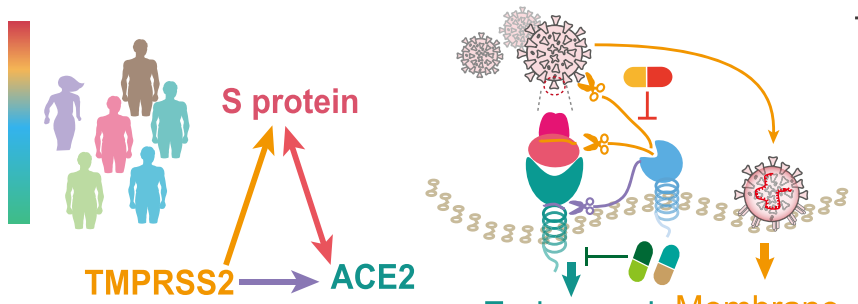

Endosomal Membrane pathway fusion

TMPRSS2 polymorphisms or dysregulation

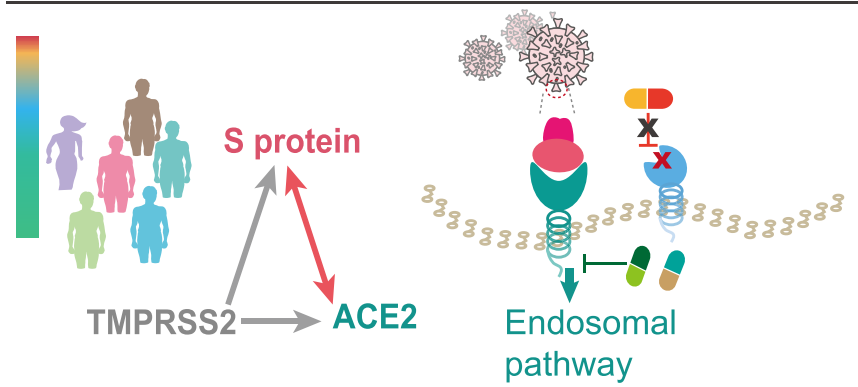

Therapeutic options:

○Endosomal pathway

E-64D

Cathepsin inhibitors

Hydroxychloroquine or Chloroquine $\mathrm{pH} \uparrow$

E-64D

Cathepsin inhibitors

Hydroxychloroquine or Chloroquine $\mathrm{pH} \uparrow$

○TMPRSS2

Camostat mesylate

○Endosomal pathway

\section{ACE2 polymorphisms or dysregulation}

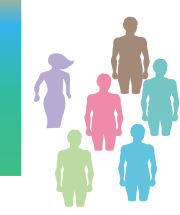

S protein

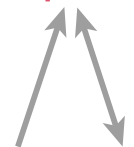

TMPRSS2 $\longrightarrow$ ACE2

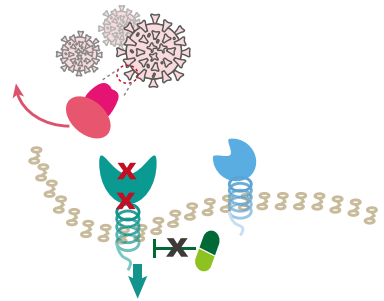

Endosomal pathway
SARS-CoV-2 Severity

Fatal Severe Mild Asymptomatic

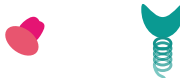

Fig. 2 (See legend on next page.) 
(See figure on previous page.)

Fig. 2 Structural view of the coding-region variants in ACE2 and TMPRSS2 and a proposed pharmacogenomics model of effective combination therapies for COVID-19. a Full-length structures of the sodium-dependent neutral amino acid transporter B(0)AT1 (SLC6A19, red)-ACE2 (blue) heterodimer in its homodimeric form complexed with the receptor binding domain (RBD, mint) of SARS-CoV-2 (PDB ID: 6M17). Highly deleterious variants are labeled as yellow spheres on ACE2. Insets depict mutations in residues 383 through 427 (top) and residues 708 through 731 (bottom). b Homology model of the catalytic chain (256-492) of TMPRSS2. Highly deleterious mutations are labeled as yellow spheres. c A proposed model of effective combination therapies (i.e., hydroxychloroquine, E-64D (a protease inhibitor), and camostat mesylate (an approved TMPRSS2 for treatment of chronic pancreatitis in Japan)) for COVID-19 by blocking ACE2 and TMPRSS2 across different populations with three genotypes. Relationship among spike (S) protein of SARS-CoV-2, ACE2, and TMPRSS2 were shown as a triangle, with each pair connecting by physical binding (double-headed arrow) or cleavage (single-headed arrow). We propose three hypotheses for COVID-19 therapeutic options: (i) for patients with wild-type or naïve expression of ACE2 and TMPRSS2, hydroxychloroquine (or chloroquine, or E-64D) combined with camostat may offer more clinical benefit; (ii) for patients with polymorphisms or dysregulation on TMPRSS2, hydroxychloroquine or chloroquine monotherapy may offer more clinical benefit; and (iii) for patients with polymorphisms or dysregulation on ACE2, the patients who might have mild symptoms can recover in a short period. All three pharmacogenomics models for COVID-19 must be validated both experimentally and clinically before being used in patients

observation suggests that developmental regulation of TMPRSS2 may link the relative protection of infants and children from COVID-19. Thus, it should be of great interest to investigate the age-related polymorphisms for TMPRSS2, such as using the Genetic Epidemiology Research on Adult Health and Aging (GERA) cohort [18], in the future.

\section{Host genetic factors guide personalized treatment of COVID-19}

There are currently no approved effective medications against COVID-19. Several national and international research groups are working on the development of vaccines to prevent COVID-19, but effective vaccines not likely to be available for many months. Several potentially repurposable drugs (Fig. 2c), including melatonin [19], hydroxychloroquine, and chloroquine, are under investigation for treatment of COVID-19 [20]. A primary mechanism-of-action of hydroxychloroquine and chloroquine is to inhibit virus entry by targeting the endosomal pathway [20]. Hydroxychloroquine and chloroquine is known to increase the $\mathrm{pH}$ of endosomes, which inhibits membrane fusion, a required mechanism for viral entry into the cell [21]. Additionally, inhibition of SARS-CoV2 could be due to differential glycosylation of both ACE2 and the spike protein [21]. As shown in Fig. 1b, several variants identified in the AFR and AMR populations, including p.Met383Thr, p.Pro389His, and p.Asp427Tyr (the pathogenic variants in ACE2 slightly inhibit interaction with the $\mathrm{S}$ protein), may influence the clinical efficacy of hydroxychloroquine or chloroquine. This may help explain why treatment of hydroxychloroquine was not significantly associated with difference in in-hospital mortality [22]. However, further pharmacogenomic studies that integrate drug response and genetic data from patients with COVID-19 are urgently needed.

In addition to the endosomal pathway, fusion of viral and host cellular membranes through $\mathrm{S}$ protein conformational changes is another way for coronavirus entry into the host cell [23]. This process can be blocked by a TMPRSS2 inhibitor (camostat mesylate, a drug approved in Japan) [5]. The mechanisms whereby TMPRSS2 promotes cellular entry of SARS-CoV-2 can be summarized by two aspects based on its proteolytic function (Fig. 2). The first is $\mathrm{S}$ protein cleavage at $\mathrm{S} 1 / \mathrm{S} 2$ and S2' sites, which might be the reason why SARS$\mathrm{CoV}-2$ entry into cells depends on TMPRSS2. The infection and pathogenesis of SARS-CoV-2 depends on the presence of TMPRSS2, in the face of the cellular elevated $\mathrm{pH}$ environment $[5,24,25]$. The inhibitors of endosomal acidification such as $\mathrm{CatB} / \mathrm{L}$ inhibitor E-64D and hydroxychloroquine/chloroquine may only work for TMPRSS2-absence patients who are infected by SARSCoV-2, and may have less effect or no effect for the patients with wild-type of TMPRSS2 [5, 24]. Therefore, the EUR and AFR populations might be more sensitive to hydroxychloroquine or chloroquine by carrying missense variants and stop-gained variants on TMPRSS2 (Figs. 1c and 2c). Yet, for patients who have wild-type of ACE2 and TMPRSS2, a combination of camostat with hydroxychloroquine or chloroquine may have better clinical benefit. However, all discussed treatment strategies must be validated by randomized controlled trials before clinical use. The second mechanism is cleavage of ACE2 by TMPRSS2 at Arginine 697 to 716 [12], which enhances viral uptake. Thus, the EUR population with p.Arg708Trp, p.Arg710Cys, p.Arg710His, and p.Arg716Cys variants in ACE2 may have mild symptoms after SARS-CoV-2 infection as ACE2 loses the cleavage site by TMPRSS2 and changes the ACE2 dimer formation [26] (Fig. 2c).

\section{Discussion and future directions: call for host genetics initiative for COVID-19}

A few limitations merit consideration. Current analysis examined massive genomic data from general population, not COVID-19 patient-specific populations. All genetic associations identified in current study are 
urgently needed to be tested in COVID-19 patients in the near future. As the high-resolution protein structure of TMPRSS2 is not yet available, further functional observations and clinical validation are warranted for all abovementioned genetic and pharmacogenomics findings. We anticipate that large-scale genome-wide association studies (GWAS) are urgently needed to identify likely causal host genetic risk factors for severe COVID19 outcomes using genetic data from patients with COVID-19; such knowledge will improve risk stratification of individuals exposed to or testing positive for SARS-CoV-2 and allow for precision medicine interventions for COVID-19. A COVID-19 host genetics initiative is already underway to bring together the human genetics research community to generate, share, and analyze data in a search for the genetic determinants of COVID-19 susceptibility, severity, and outcomes [27]. The first COVID-19 GWAS identified the 3p21.31 gene cluster (including SLC6A20, LZTFL1, CCR9, FYCO1, CXCR6, and XCR1) as a genetic susceptibility locus in severe patients with COVID-19 and respiratory failure [28]. Yet, our study aims to look for SNPs associated with disease severity of COVID-19, but not disease susceptibility. In summary, systematic identification of the genetic determinants of COVID-19 susceptibility, severity, and clinical outcome, including both virus and host factors (e.g., ACE2 and TMPRSS2 polymorphisms), could guide personalized treatment in the emerging COVID19 pandemic and even explain current epidemiologic observations (i.e., males, elderly at high risk, and clinical comorbidities) and natural history.

\section{Conclusions}

This comprehensive comparative genetic analysis of approximately 81,000 human genomes suggested possible associations of ACE2 and TMPRSS2 DNA polymorphisms with COVID-19 susceptibility, severity, and clinical outcomes. We found that ACE2 polymorphisms were more likely to be associated with cardiovascular and pulmonary conditions by altering the angiotensinogen-ACE2 interactions, such as p.Arg514Gly in the African/African-American population. Unique but prevalent polymorphisms in TMPRSS2, including p.Val160Met (rs12329760), may provide potential explanations for differential genetic susceptibility to COVID19 as well as for risk factors, including cancer and the high-risk group of male patients. We highlighted that polymorphisms in ACE2 or TMPRSS2 could guide personalized treatments (i.e., hydroxychloroquine and camostat) for COVID-19. In summary, this study suggested that ACE2 or TMPRSS2 DNA polymorphisms were likely associated with genetic susceptibility to COVID-19, which calls for a human genetics initiative for fighting the COVID-19 pandemic.

\section{Abbreviations}

1KGP: 1000 Genomes Project; ACE2: Angiotensin-converting enzyme 2; CoV: Coronavirus; COVID-19: Coronavirus Disease 2019; eQTL: Expression quantitative trait loci; gnomAD: Genome Aggregation Database;

MERS: Middle East respiratory syndrome; SARS: Severe acute respiratory syndrome; SARS-CoV-2: Severe acute respiratory syndrome coronavirus 2; ESP: Exome Sequencing Project; S: Spike; TMPRSS2: Transmembrane serine protease 2

\section{Acknowledgements}

We thank all helpful discussions and critical comments regarding this manuscript from the COVID-19 Research Intervention Advisory Committee members at the Cleveland Clinic. SCE is the Alfred Lerner Memorial Chair of Innovative Research and CE is the Sondra J. and Stephen R. Hardis Endowed Chair of Cancer Genomic Medicine at the Cleveland Clinic.

\section{Authors' contributions}

F.C. conceived the study. Y.H., J.Z., and W.M. performed all experiments and data analysis. A.K., M.K.C, N.S., L.J., C.E., and S.E. discussed and interpreted all results. F.C., Y.H., C.E., and S.E. wrote and critically revised the manuscript with contributions from other co-authors. All authors read and approved the final manuscript.

\section{Funding}

This work was supported by the National Heart, Lung, and Blood Institute of the National Institutes of Health $(\mathrm{NIH})$ under Award Number ROOHL138272 and the National Institute of Aging under Award Number R01AG066707 to F.C. This work was supported, in part, by the VeloSano Pilot Program (Cleveland Clinic Taussig Cancer Institute).

\section{Availability of data and materials}

All population genetic data used in this study are free and available at three databases: (i) Genome Aggregation Database (gnomAD v3: gnomad. broadinstitute.org, covering 9 geographical areas), (ii) Exome Sequencing Project (ESP: evs.gs.washington.edu/EVS/), and (iii) 1000 Genomes Project (1KGP, www.internationalgenome.org).

Ethics approval and consent to participate

Not applicable.

Consent for publication

Not applicable.

\section{Competing interests}

The content of this publication does not necessarily reflect the views of the Cleveland Clinic. The authors declare no competing interests.

\section{Author details}

${ }^{1}$ Genomic Medicine Institute, Lerner Research Institute, Cleveland Clinic, Cleveland, OH 44195, USA. ${ }^{2}$ Department of Systems Biology and Department of Biomedical Informatics, Herbert Irving Comprehensive Center, Columbia University, New York, NY 10032, USA. ${ }^{3}$ Department of Molecular Medicine, Cleveland Clinic Lerner College of Medicine, Case Western Reserve University, Cleveland, OH 44195, USA. ${ }^{4}$ Department of Cardiovascular Medicine, Heart, Vascular and Thoracic Institute, Cleveland Clinic, Cleveland, OH 44195, USA. ${ }^{5}$ Lerner Research Institute, Cleveland Clinic, Cleveland, OH 44195, USA.

${ }^{6}$ Department of Genetics and Genome Sciences, School of Medicine, Case Western Reserve University, Cleveland, OH 44106, USA. ${ }^{7}$ Case Comprehensive Cancer Center, School of Medicine, Case Western Reserve University,

Cleveland, $\mathrm{OH}$ 44106, USA.

Received: 25 April 2020 Accepted: 22 June 2020

Published online: 15 July 2020

\section{References}

1. Ashour HM, Elkhatib WF, Rahman MM, Elshabrawy HA. Insights into the recent 2019 novel coronavirus (SARS-CoV-2) in light of past human coronavirus outbreaks. Pathogens. 2020;9(3):186.

2. Dong $E$, Du H, Gardner L. An interactive web-based dashboard to track COVID-19 in real time. Lancet Infect Dis. 2020;20(5):533-4. 
3. Dong Y, Mo X, Hu Y, Qi X, Jiang F, Jiang Z, Tong S. Epidemiology of COVID19 among children in China. Pediatrics. 2020;8(6):2118-20.

4. Lek M, Karczewski KJ, Minikel EV, Samocha KE, Banks E, Fennell T, O'DonnellLuria AH, Ware JS, Hill AJ, Cummings BB, et al. Analysis of protein-coding genetic variation in 60,706 humans. Nature. 2016;536(7616):285-91.

5. Hoffmann M, Kleine-Weber H, Schroeder S, Kruger N, Herrler T, Erichsen S, Schiergens TS, Herrler G, Wu NH, Nitsche A, et al. SARS-CoV-2 cell entry depends on ACE2 and TMPRSS2 and is blocked by a clinically proven protease inhibitor. Cell. 2020;181(2):271-80.

6. Stopsack KH, Mucci LA, Antonarakis ES, Nelson PS, Kantoff PW. TMPRSS2 and COVID-19: serendipity or opportunity for intervention? Cancer Discov. 2020; 10(6):779-82.

7. Zou X, Chen K, Zou J, Han P, Hao J, Han Z. Single-cell RNA-seq data analysis on the receptor ACE2 expression reveals the potential risk of different human organs vulnerable to 2019-nCoV infection. Front Med. 2020. https:// doi.org/10.1007/s1 1684-020-0754-0.

8. Guo T, Fan Y, Chen M, Wu X, Zhang L, He T, Wang H, Wan J, Wang X, Lu Z. Cardiovascular implications of fatal outcomes of patients with coronavirus disease 2019 (COVID-19). JAMA Cardiol. 2020. https://doi.org/10.1001/ jamacardio.2020.1017.

9. Wang K, Li M, Hakonarson H. ANNOVAR: functional annotation of genetic variants from high-throughput sequencing data. Nucleic Acids Res. 2010; 38(16):e164.

10. Li W, Zhang C, Sui J, Kuhn JH, Moore MJ, Luo S, Wong SK, Huang IC, Xu K, Vasilieva $\mathrm{N}$, et al. Receptor and viral determinants of SARS-coronavirus adaptation to human ACE2. EMBO J. 2005;24(8):1634-43.

11. Kuster GM, Pfister O, Burkard T, Zhou Q, Twerenbold R, Haaf P, Widmer AF, Osswald S. SARS-CoV2: should inhibitors of the renin-angiotensin system be withdrawn in patients with COVID-19? Eur Heart J. 2020;41(19):1801-3.

12. Heurich A, Hofmann-Winkler H, Gierer S, Liepold T, Jahn O, Pohlmann S. TMPRSS2 and ADAM17 cleave ACE2 differentially and only proteolysis by TMPRSS2 augments entry driven by the severe acute respiratory syndrome coronavirus spike protein. J Virol. 2014;88(2):1293-307.

13. Consortium GT. Human genomics. The Genotype-Tissue Expression (GTEx) pilot analysis: multitissue gene regulation in humans. Science. 2015; 348(6235):648-60.

14. Zheng Z, Huang D, Wang J, Zhao K, Zhou Y, Guo Z, Zhai S, Xu H, Cui H, Yao $\mathrm{H}$, et al. QTLbase: an integrative resource for quantitative trait loci across multiple human molecular phenotypes. Nucleic Acids Res. 2020;48(D1): D983-91.

15. Shirato K, Kawase M, Matsuyama S. Wild-type human coronaviruses prefer cell-surface TMPRSS2 to endosomal cathepsins for cell entry. Virology. 2018; 517:9-15.

16. Yu J, Ouyang W, Chua MLK, Xie C. SARS-CoV-2 transmission in patients with cancer at a tertiary care hospital in Wuhan. China JAMA Oncol. 2020. https://doi.org/10.1001/jamaoncol.2020.0980.

17. Schuler A, Habermann C, Plosa J, et al. Age-related expression of SARS-CoV2 primining protease TMPRSS2 in the developing lung. 2020. https://doi. org/10.1101/2020.05.22.111187 bioRxiv preprint doi: https://doi.org/10.1101/ 2020.05.22.111187

18. Mostafavi H, Berisa T, Day FR, Perry JRB, Przeworski M, Pickrell JK. Identifying genetic variants that affect viability in large cohorts. PLoS Biol. 2017;15(9): e2002458.

19. Zhou Y, Hou Y, Shen J, Huang Y, Martin W, Cheng F. Network-based drug repurposing for novel coronavirus 2019-nCoV/SARS-CoV-2. Cell Discov. 2020; 6:14.

20. Sanders JM, Monogue ML, Jodlowski TZ, Cutrell JB. Pharmacologic treatments for coronavirus disease 2019 (COVID-19): a review. JAMA. 2020. https://doi.org/10.1001/jama.2020.6019.

21. Savarino A, Di Trani L, Donatelli I, Cauda R, Cassone A. New insights into the antiviral effects of chloroquine. Lancet Infect Dis. 2006;6(2):67-9.

22. Rosenberg ES, Dufort EM, Udo T, Wilberschied LA, Kumar J, Tesoriero J, Weinberg P, Kirkwood J, Muse A, DeHovitz J, et al. Association of treatment with hydroxychloroquine or azithromycin with in-hospital mortality in patients with COVID-19 in New York state. JAMA. 2020. https://doi.org/10. 1001/jama.2020.8630.

23. Walls AC, Tortorici MA, Snijder J, Xiong X, Bosch BJ, Rey FA, Veesler D. Tectonic conformational changes of a coronavirus spike glycoprotein promote membrane fusion. Proc Natl Acad Sci U S A. 2017;114(42):1115762.
24. Shulla A, Heald-Sargent T, Subramanya G, Zhao J, Perlman S, Gallagher T. A transmembrane serine protease is linked to the severe acute respiratory syndrome coronavirus receptor and activates virus entry. J Virol. 2011;85(2): 873-82.

25. Simmons G, Gosalia DN, Rennekamp AJ, Reeves JD, Diamond SL, Bates P. Inhibitors of cathepsin L prevent severe acute respiratory syndrome coronavirus entry. Proc Natl Acad Sci U S A. 2005;102(33):11876-81.

26. Yan R, Zhang Y, Li Y, Xia L, Guo Y, Zhou Q. Structural basis for the recognition of SARS-CoV-2 by full-length human ACE2. Science. 2020; 367(6485):1444-8,

27. Initiative C-HG. The COVID-19 host genetics Initiative, a global initiative to elucidate the role of host genetic factors in susceptibility and severity of the SARS-CoV-2 virus pandemic. Eur J Hum Genet. 2020;28(6):715-8.

28. Group TSC-G. Genomewide association study of severe Covid-19 with respiratory failure. N Engl J Med. 2020. https://doi.org/10.1056/ NEJMoa2020283.

\section{Publisher's Note}

Springer Nature remains neutral with regard to jurisdictional claims in published maps and institutional affiliations.
Ready to submit your research? Choose BMC and benefit from:

- fast, convenient online submission

- thorough peer review by experienced researchers in your field

- rapid publication on acceptance

- support for research data, including large and complex data types

- gold Open Access which fosters wider collaboration and increased citations

- maximum visibility for your research: over $100 \mathrm{M}$ website views per year

At $\mathrm{BMC}$, research is always in progress.

Learn more biomedcentral.com/submissions 